\title{
La problematica della compliance nella gestione dell'iperfosfatemia
}

\author{
Carlo Alfieri, Piergiorgio Messa
}

Area Omogenea di Nefrologia, Dialisi e Trapianto Renale, Fondazione IRCCS Ca' Granda Ospedale Maggiore Policlinico, Milano

\begin{abstract}
Compliance issues in the management of hyperphosphatemia
Chronic kidney disease (CKD) is an important problem in public health and has significant morbidity and mortality implications. Mineral and bone disorders are common during CKD and occur in the early stages of the disease. During CKD a progressive reduction of the excretion of many substances, including phosphorus, is reported. The phosphorus balance is altered from the early stages of disease, and its variation has been related both to mineral bone disorders and cardiovascular disease. Of note, phosphate levels, which are frequently high in late stages of CKD, do not represent the total phosphate balance, which is influenced also by dietary phosphate intake, intestinal absorption and renal excretion.

Correct phosphate control in CKD patients can be obtained by operating at different levels: 1) optimization of dialysis: type of dialysis (hemodialysis versus peritoneal dialysis), vascular access characteristics, modalities and duration of dialysis; 2 ) reduction of dietary phosphate intake, obtained by the consumption of low-phosphate food and by educating patients in cooking styles; 3 ) use of drugs able to reduce intestinal phosphate absorption. For each of these approaches, and to ameliorate patient compliance, it is mandatory to correctly instruct patients, explaining to them specific concepts about hyperphosphatemia and its treatment and about the use of phosphate binders.

The aim of this review is to summarize the principal concepts concerning phosphate anomalies during CKD and compliance difficulties in the treatment of hyperphosphatemia.
\end{abstract}

Keywords: Compliance, Dialysis, Nutrition, Phosphorus balance, Phosphate binders

\section{Introduzione}

La malattia renale cronica (CKD) rappresenta oggi un problema importante di salute pubblica, considerati la vasta prevalenza e incidenza e l'alto grado di mortalità e morbilità ad essa correlati (1). La progressiva perdita della funzione renale si associa alla ritenzione di numerose sostanze escrete dai reni in condizioni fisiologiche, tra cui il fosforo (2). II corretto controllo del bilancio fosforico è uno dei tanti aspetti che richiedono una particolare attenzione nel trattamento di questi pazienti, tanto nelle fasi iniziali quanto nelle fasi avanzate di

Accepted: August 30, 2016

Published online: September 7, 2016

Indirizzo per la corrispondenza:

Prof. Piergiorgio Messa

Area Omogenea di Nefrologia, Dialisi e Trapianto Renale

Fondazione IRCCS Cà Granda Ospedale Maggiore Policlinico

Via della Commenda 15

20122 Milano

pmessa@policlinico.mi.it malattia (3). Nello stadio terminale di malattia, poi, questo problema raggiunge il suo impatto maggiore poiché l'unica fonte di rimozione del fosforo è sovente rappresentata dalla dialisi, che da sola risulta essere però spesso insufficiente.

I dati DOPPS (Dialysis Outcomes and Practice Patterns Study), raccolti su una coorte di più di 20.000 pazienti sottoposti a trattamento emodialitico cronico hanno dimostrato che, nonostante la terapia medica, circa il $30 \%$ dei pazienti emodializzati presenta livelli sierici elevati di fosforo $(>5,5 \mathrm{mg} / \mathrm{dL})(4)$.

Un corretto controllo del fosforo sembrerebbe avere implicazioni importanti nell'influenzare la progressione della malattia renale, la formazione di calcificazioni vascolari e di conseguenza la mortalità generale e cardiovascolare. Bisogna segnalare però che la quasi totalità degli studi in quest'ambito sono di natura retrospettiva o osservazionale, e non di intervento. Tra questi segnaliamo un lavoro retrospettivo pubblicato nel 2005 da Kestenbaum et al, effettuato su 3490 pazienti affetti da CKD, in cui è stato dimostrato un incremento del rischio di morte per cause generali per livelli sierici di fosforo $>3,5 \mathrm{mg} / \mathrm{dL}$, con un incremento lineare per ogni aumento di $0,5 \mathrm{mg} / \mathrm{dL}$ di fosforemia. Gli elevati livelli di fosforo, 
inoltre, sono risultati indipendentemente associati all'incremento della mortalità (5).

Il controllo del fosforo nei pazienti affetti da malattia renale cronica andrebbe ottenuto agendo su diversi aspetti terapeutici e comportamentali, sempre e comunque con la totale collaborazione da parte del paziente. Tutto ciò evidentemente presuppone una consapevolezza del paziente degli interventi prescritti, e soprattutto del motivo per cui essi sono stati raccomandati.

La sensibilizzazione dei pazienti potrebbe essere riassunta in due grandi punti, la cui caratterizzazione sarà l'argomento principale di cui tratterà questo lavoro:

1) La sensibilizzazione sulla consapevolezza di alcuni concetti specifici riguardanti l'iperfosforemia e il suo trattamento.

2) La sensibilizzazione sull'importanza della corretta assunzione terapeutica dei farmaci chelanti il fosforo.

\section{Concetti specifici riguardanti l'iperfosforemia e il suo trattamento}

Il trattamento dell'iperfosforemia, ancora prima che farmacologico, dovrebbe prevedere l'ottimizzazione della terapia dialitica (negli stadi terminali di malattia) e dell'alimentazione.

Solo nel momento in cui i due sia la terapia dialitica sia l'alimentazione non permettono un corretto controllo del fosforo, evento purtroppo piuttosto frequente, è necessario agire con la terapia farmacologica che come vedremo andrà individualizzata per ogni singolo paziente.

\section{Terapia dialitica}

L'ottimizzazione della terapia dialitica, sia essa emodialitica o dialitica peritoneale dovrebbe essere preliminare a qualsiasi intervento medico correttivo più specifico.

In emodialisi è necessario avere a disposizione un accesso vascolare correttamente funzionante, possibilmente nativo. Una valutazione periodica del corretto funzionamento dell'accesso dovrebbe essere sempre eseguita. In aggiunta, la scelta della metodica dialitica e la durata delle sedute emodialitiche sono fattori altrettanto importanti (6). Diversi studi presenti in letteratura hanno infatti dimostrato una miglior capacità di rimozione del fosforo a favore delle metodiche online rispetto alla bicarbonato-emodialisi convenzionale (7), caratteristica che potrebbe in parte influenzare la maggior sopravvivenza riscontrata nei pazienti sottoposti a metodiche on-line rispetto a quelli trattati con bicarbonato-emodialisi. Ciononostante, da sole queste metodiche maggiormente efficienti non sembrerebbero essere sufficienti a raggiungere un bilancio di fosforo neutro e quindi da sole non adeguate al trattamento dell'iperfosforemia. La rimozione del fosforo è però anche sensibilmente influenzata dalla durata del trat- tamento sostitutivo. In un lavoro pubblicato nel 2008, in cui si valutava l'impatto della durata della dialisi sulla rimozione delle tossine uremiche, è stato dimostrato un incremento direttamente proporzionale della quota di rimozione del fosforo con la durata della singola seduta emodialitica (8). Questo concetto è molto importante, considerando che spesso sono proprio i pazienti a richiedere una riduzione dei tempi di trattamento.

Per quanto riguarda la dialisi peritoneale, non attuabile nella totalità dei pazienti, diversi studi hanno riportato una sua minor capacità di rimozione del fosforo rispetto alla emodialisi. Come per l'emodialisi, nella dialisi peritoneale la quota di fosforo rimossa è strettamente dipendente dalla quota iniziale e dai volumi di scambio. La presenza però di una diuresi residua, che permette l'escrezione urinaria di una minima quota di fosforo, associata alla caratteristica "continuità" del trattamento dialitico peritoneale permette di osservare una minor incidenza di iperfosfatemia nei pazienti sottoposti a questo tipo di trattamento rispetto ai pazienti in emodialisi (9).

\section{Significato clinico del controllo del fosforo e importanza del bilancio del fosforo}

Una capillare istruzione del paziente, fin dalle prime fasi di malattia renale sul significato clinico del controllo del fosforo ha una grande importanza. I pazienti devono essere resi consci dell'importanza che riveste un corretto controllo dei valori di fosforemia non solo per il metabolismo minerale e per la salute dell'osso, ma anche per il suo impatto sulla mortalità. Questo purtroppo non è sempre semplice, come dimostrato in un recente lavoro condotto su 153 pazienti emodializzati da Shutto et al, nel quale è stato riportato che circa il 78\% dei pazienti non era a conoscenza dei rischi legati al consumo di cibi e bevande ad alto contenuto fosforico (10). Quanto detto potrebbe risultare in contrasto con alcuni dati riportati in letteratura riguardanti il rapporto diretto tra livelli di fosforemia, progressione della malattia renale e mortalità nel lungo termine. Recenti studi di natura osservazionale hanno infatti mostrato risultati contrastanti, non riportando differenze di sopravvivenza in soggetti con diversi livelli di fosforo. In un lavoro pubblicato nel 2013 da Mehrotra et al, in cui venivano riportati i risultati di una analisi effettuata su più di 10000 pazienti con un filtrato $60 \mathrm{~mL} / \mathrm{min}$ seguiti per un tempo medio di follow-up di 2 anni e suddivisi in quattro gruppi sulla base dei livelli di fosforemia non è emersa nessuna associazione indipendente tra i livelli di fosforo sierico, il rischio di progressione della malattia renale e il rischio di morte (evento riscontrato in 578 pazienti) (11).

L'assenza di relazioni certe potrebbe essere da imputare al fatto che il valore di fosforemia non rappresenterebbe un chiaro riflesso del bilancio fosforico totale. Ciò è anche suggerito da alcuni studi in cui viene indicato come il livello di fosforo nel sangue non mostri relazione né con la quota di fosforo 
assunta dalla dieta né con quella escreta con le urine, a loro volta però correlate con la quantità di fosforo assorbita a livello intestinale (12). Questo è confermato anche dal riscontro di una modesta riduzione dei livelli di fosforemia rilevata nei pazienti nei quali viene attuata una stretta restrizione dell'assunzione di fosforo, a fronte di una proporzionale riduzione dell'escrezione urinaria di fosforo (13).

L'associazione tra aumentato apporto di fosforo con la dieta e rischio cardiovascolare è stato anch'esso indagato in studi prevalentemente osservazionali. L'eccessivo apporto di fosforo con la dieta sembrerebbe incidere negativamente sulla struttura e la funzionalità cardiaca e sulla frequenza degli eventi cardiovascolari, anche in assenza di malattia renale. Nel lavoro pubblicato da Yamamoto et al nel 2013, in cui è stata esplorata in 4494 pazienti l'associazione tra assunzione di fosforo con la dieta e massa ventricolare sinistra valutata attraverso risonanza magnetica, è stata riscontrata, soprattutto nel sesso femminile, un'associazione tra un elevato intake di fosforo con la dieta e presenza di ipertrofia ventricolare sinistra (14). In un altro lavoro, pubblicato nello stesso anno da Palomino et al, veniva dimostrato un incremento significativo del rischio di eventi cardiovascolari all'aumentare dell'escrezione urinaria di fosforo che, come detto, può essere considerato un indicatore indiretto dell'introito dietetico del minerale (15). L'educazione del paziente quindi in questo senso viene al primo posto, e ha un ruolo predominante. Questa però è resa molto difficoltosa dal fatto che nella maggior parte dei casi i pazienti focalizzano la loro attenzione non tanto sul bilancio del fosforo quanto sui livelli di fosforemia. A complicare ulteriormente il quadro, non possiamo non considerare che tutti i presidi terapeutici oggi a disposizione del medico nefrologo sono finalizzati principalmente o esclusivamente alla correzione dei livelli di fosforemia piuttosto che alla modificazione del bilancio del fosforo.

\section{Quali sono le reali sorgenti del fosforo che si introduce?}

Il contenuto di fosforo presente negli alimenti e la "non tossicità" del fosforo aggiunto è un argomento largamente dibattuto in letteratura da diverso tempo. I fosfati vengono aggiunti ai cibi per diversi motivi: prevenire grumi negli impasti, come agenti lievitanti (fosfati di sodio), migliorare o modificare il sapore e la consistenza del cibo (polifosfati nei preparati a base di carne). Possono essere usati per dare un gusto particolare (acido fosforico nella cola); inoltre sono utili per mantenere più a lungo gli alimenti riducendo i processi di ossidazione dei lipidi.

Già nel 1973 la Food and Drug Administration tentò di classificare gli additivi a base di fosfato dei cibi, definendoli come "non dannosi", in quanto ininfluenti sia sulla natura che sul valore nutrizionale stesso dei cibi. Nel 1988, inoltre la stessa Organizzazione propose di non includere nelle informazioni nutrizionali dei prodotti il contenuto di fosfato poiché esso non aveva nessun impatto sulla salute pubblica.
Negli stessi anni, però la comunità scientifica, e in particolare Bell et al dimostravano come una dieta ad alto contenuto di fosforo inducesse un incremento nei livelli sierici e urinari di fosforo (16).

Al giorno d'oggi viene posta molta attenzione all'educazione dei pazienti malati di rene, in particolare dei pazienti dializzati. Indipendentemente dall'assunzione di terapie per l'iperfosforemia, è fondamentale che i pazienti siano coscienti e in grado di gestire l'assunzione orale di fosforo e vengano trattati nel modo più efficiente possibile. L'utilizzo di opuscoli, accessibili a tutti i pazienti e presentati in un formato chiaro potrebbe essere un modo facile e intuitivo di istruzione. A questo però bisognerebbe aggiungere una preparazione diretta del paziente stesso, che spesso si trova in difficoltà per ragioni sociali e di compliance personale. II paziente dovrebbe anche essere istruito sui diversi procedimenti di cottura dei cibi (ad esempio, la bollitura) e sulla necessità di evitare cibi in cui vi sia un'alta quota di fosfati aggiunti, in quanto questi fosfati hanno una maggiore assorbibilità di quelli naturalmente presenti nei cibi (17). Quest'ultimo concetto è molto importante, basti infatti pensare che una piccolissima parte della popolazione affetta da malattia renale cronica è a conoscenza di quali siano i cibi a maggior contenuto di fosforo. Ciò è stato in parte provato in un recente lavoro, in cui è stato mostrato attraverso una survey effettuata su pazienti affetti da malattia renale cronica, che la percentuale di pazienti a conoscenza del contenuto di fosforo della loro dieta era significativamente minore della percentuale di pazienti a conoscenza del contenuto di glucosio.

Pochissima considerazione inoltre viene data al quantitativo di fosforo assunto dai pazienti da fonti alternative, che possono essere rappresentati da supplementi dietetici e/o farmaci il cui contenuto di fosforo, è presente soprattutto sotto forma di eccipienti (18).

Infine, ma non ultimo per importanza, l'istituzione di un servizio di consultazione nutrizionale di facile accessibilità ai pazienti potrebbe in alcuni casi fornire ulteriori indicazioni e rappresentare una guida per i pazienti anche a lungo termine.

\section{Fattori legati all'utilizzo di chelanti}

A partire dagli anni Settanta del secolo scorso, l'avvento in commercio di farmaci in grado di ridurre l'assorbimento intestinale di fosforo ha permesso un miglior controllo dell'iperfosforemia nei pazienti affetti da malattia renale cronica. Nonostante siano ad oggi numerosi i farmaci a disposizione del clinico, il chelante del fosforo ideale, caratterizzato da una efficacia ottimale, da una buona tolleranza, da una minima tossicità sia in termini di accumulo che in termini di interazione con altri farmaci, e che sia caratterizzato da un ottimo rapporto costo-efficacia, al momento non esiste. Malgrado ciò, la presenza in commercio di diverse formulazioni permette una personalizzazione della terapia chelante il fosforo.

Non essendo disponibili attualmente linee guida chiare 
sull'utilizzo di questi farmaci e soprattutto sulla scelta del chelante da impiegare, è necessario che il clinico ne moduli l'utilizzo sulla base delle caratteristiche specifiche dei farmaci e dei singoli pazienti che ne richiedono l'utilizzo.

Sicuramente il contenuto e l'assorbimento del minerale contenuto nel chelante (calcio, alluminio, lantanio) rappresentano un fattore chiave nella scelta del chelante. In questo senso, la presenza oggi in commercio di farmaci chelanti il fosforo senza contenuto di calcio, non gravati quindi da problemi di accumulo permette anche l'associazione di più farmaci chelanti in un solo paziente e quindi la riduzione anche di quelli a contenuto minerale.

Sul versante del paziente, la valutazione delle comorbilità, come ad esempio la presenza di calcificazioni vascolari e/o ipercalcemia, la tolleranza del paziente alle terapie e l'aspettativa di vita sono fattori da considerare. La quantità di pillole che il paziente assume è un altro fattore determinante, in quanto direttamente correlato alla aderenza terapeutica dei pazienti (19).

In ultima analisi, ma non meno importante, è rilevante tenere bene conto del rapporto costo-efficacia di ogni singolo farmaco.

\section{Conclusioni}

Il controllo del bilancio del fosforo è un aspetto importante nella gestione del paziente affetto da malattia renale cronica, e dovrebbe essere concretizzato già nelle fasi iniziali di nefropatia. L'utilizzo di farmaci inibenti l'assorbimento intestinale di fosforo e l'ottimizzazione della terapia dialitica rappresentano due punti importanti nella gestione di questi pazienti che però non possono prescindere da una corretta istruzione dei pazienti sull'alimentazione, volta non solo a insegnare come ridurre l'apporto di fosforo con la dieta, ma anche e soprattutto a illustrarne l'importanza clinica sia per la malattia renale in sé che per le patologie cardiovascolari.

Una maggior presa di coscienza del problema, anche da parte del nefrologo, permetterebbe quindi di aumentare in maniera significativa l'accettazione del problema e l'aderenza terapeutica dei pazienti.

\section{Disclosures}

Financial support: This Supplement has been supported by an unrestricted educational grant provided by Vifor Fresenius Medical Care Renal Pharma Italia S.r.l.

Conflict of interest: The authors declare they do not have any conflict of interest.

\section{Bibliografia}

1. Nwankwo E, Bello AK, El Nahas AM. Chronic kidney disease: stemming the global tide. Am J Kidney Dis. 2005;45(1):201-8.

2. Hruska KA, Mathew S, Lund R, Qiu P, Pratt R. Hyperphosphatemia of chronic kidney disease. Kidney Int. 2008;74(2):148-57.

3. Russo D, Bellasi A, Pota A, Russo L, Di lorio B. Effects of phosphorus-restricted diet and phosphate-binding therapy on outcomes in patients with chronic kidney disease. J Nephrol. 2015;28(1):73-80.

4. Serum phosphate (national sample) US DOPPS December 2015. www.DOPPS.org

5. Kestenbaum B, Sampson JN, Rudser KD, et al. Serum phosphate levels and mortality risk among people with chronic kidney disease. J Am Soc Nephrol. 2005;16(2):520-8.

6. Messa P, Cerutti R, Brezzi B, Alfieri C, Cozzolino M. Calcium and phosphate control by dialysis treatments. Blood Purif. 2009;27(4):360-8.

7. Lornoy W, De Meester J, Becaus I, Billiouw JM, Van Malderen PA, Van Pottelberge M. Impact of convective flow on phosphorus removal in maintenance hemodialysis patients. J Ren Nutr. 2006;16(1):47-53.

8. Eloot S, Van Biesen W, Dhondt A, et al. Impact of hemodialysis duration on the removal of uremic retention solutes. Kidney Int. 2008;73(6):765-70.

9. Dong J, Wang H, Wang M. Low prevalence of hyperphosphatemia independent of residual renal function in peritoneal dialysis patients. J Ren Nutr. 2007;17(6):389-96.

10. Shutto $Y$, Shimada M, Kitajima M, et al. Inadequate awareness among chronic kidney disease patients regarding food and drinks containing artificially added phosphate. PloSOne. 2013; 8(11):e78660.

11. Mehrotra R, Peralta CA, Chen SC, et al; Kidney Early Evaluation Program (KEEP) Investigators. No independent association of serum phosphorus with risk for death or progression to endstage renal disease in a large screen for chronic kidney disease. Kidney Int. 2013;84(5):989-97.

12. Noori N, Kalantar-Zadeh K, Kovesdy CP, Bross R, Benner D, Kopple JD. Association of dietary phosphorus intake and phosphorus to protein ratio with mortality in hemodialysis patients. Clin J Am Soc Nephrol. 2010;5(4):683-92.

13. Newsome B, Ix JH, Tighiouart $\mathrm{H}$, et al. Effect of protein restriction on serum and urine phosphate in the Modification of Diet in Renal Disease (MDRD) Study. Am J Kidney Dis. 2013;61(6): 1045-6.

14. Yamamoto KT, Robinson-Cohen C, de Oliveira MC, et al. Dietary phosphorus is associated with greater left ventricular mass. Kidney Int. 2013;83(4):707-14.

15. Palomino HL, Rifkin DE, Anderson C, Criqui MH, Whooley MA, Ix JH. 24-hour urine phosphorus excretion and mortality and cardiovascular events. Clin J Am Soc Nephrol. 2013;8(7):1202-10.

16. Bell RR, Draper HH, Tzeng DY, Shin HK, Schmidt GR. Physiological responses of human adults to foods containing phosphate additives. J Nutr. 1977;107(1):42-50.

17. D'Alessandro C, Piccoli G, Cupisti A. The "phosphorus pyramid": a visual tool for dietary phosphate management in dialysis and CKD patients. BMC Nephrol. 2015;16:9.

18. Calvo MS, Uribarri J. Contributions to total phosphorus intake: all sources considered. Semin Dial. 2013;26(1):54-61.

19. Chiu YW, Teitelbaum I, Misra M, de Leon EM, Adzize T, Mehrotra R. Pill burden, adherence, hyperphosphatemia, and quality of life in maintenance dialysis patients. Clin J Am Soc Nephrol. 2009;4(6):1089-96. 